Meta

Journal des traducteurs

Translators' Journal

\title{
Index des mots et sujets traités dans le volume 14
}

Volume 14, numéro 4, décembre 1969

URI : https://id.erudit.org/iderudit/003220ar

DOI : https://doi.org/10.7202/003220ar

Aller au sommaire du numéro

Éditeur(s)

Les Presses de l'Université de Montréal

ISSN

0026-0452 (imprimé)

1492-1421 (numérique)

Découvrir la revue

Citer ce document

(1969). Index des mots et sujets traités dans le volume 14. Meta, 14(4), 261-267.

https://doi.org/10.7202/003220ar d'utilisation que vous pouvez consulter en ligne.

https://apropos.erudit.org/fr/usagers/politique-dutilisation/ 


\section{INDEX DES MOTS ET SUJETS TRAITES DANS LE VOLUME 14}

A (destination), 4, p. 233.

"A bain d'huile s, 4, p. 209.

A refroidissement naturel, 4, p. 210.

A roulage direct, 3, p. 161 .

Abattoir, 3, p. 168.

Absence de correspondance, 3, p. 166.

Abréviations, 3, p. 167.

Abus de l'emprunt, 2, p. 103.

Acadien, 2, p. 110.

Accent secondaire en anglais, 2, p. 122.

Acceptation, 4, p. 210.

Accrocher, 3, p. 139

Achalandage, 4, p. 218.

Achigan, 2, p. 109.

Acoustique, 1, p. 59 .

Action, 1, p. 43.

Adaptation, 1, p. 8; 3, p. 137.

Additionneuse, 2, p. 113.

Affaires, 2, p. 113

Affectation de patriotisme, 2, p. 94

"Agent de réclamation ", 1, p. 46.

"Agent du croire », 2, p. 113.

Agriculture, 2, p. 119.

Ainsi-soit-il, 1, p. 6.

"Ajusteur d'assurance ", 1, p. 46

Ajusteur de rapport, 4, p. 210.

Aliénation linguistique, 3 , p. 178 .

Alimentation, 3, p. 167.

Alimenter, 2, p. 113

Allemand, 3, p. 169.

Ame, 1, p. 37.

Aménagement foncier, 4, p. 239.

Amplification, 4, p. 227-229.

Analyse componentielle, 4, p. 205.

Analyse et traduction, 1, p. 75 .

Analyse linguistique, 4, p. 235

Analyse par activation, 4, p. 233.

Analyse sémique, 4, p. 205.

Anatomie, 2, p. 119

Ancrer, 1, p. 37.

Anglais, 3, p. 170

Anglicisme culturel, 1, p. 11

Anglicisme en France et au Canada, 1, p. 71

Anglicisme en publicité, 3, p. 177.

Anglicismes juridiques, 1, p. 46.

Angulaire, 3, p. 139

Anguleux, 3, p. 139.

Animateur, 3, p. 146.

Animé, 4, p. 202.

Animisme, 4, p. 233.

Année d'assurance, 4, p. 244.

"Année de police ", 4, p. 244

Anthropologie, 4, p. 200.

Appétit ( $\left(l^{\prime}\right)$ vient en mangeant, 3, p. 149.

"Approche d'un problème », 2, p. 110

Arabe, 2, p. $117 ; 3$, p. $170 ; 4$, p. 249.

Archéologie, 2, p. 119.

Argot, 4, p. 247.

Arrestation, 1, p. 45.

Article, 3, p. 157 .
Askarel, 4, p. 209.

Aspect, 3, p. 139.

Assécheur, 4, p. 209.

Assemblée annuelle (STIC), 4, p. 255.

Assise, 1, p. 36

Assistant du bibliothécaire, 2, p. 113.

"Assistant-bibliothécaire ", 2, p. 114.

Assurances, 3, p. 183.

Assurances sur la vie, 4, p. 244.

Atelier, 2, p. 97.

"Attache-feuilles \&, 2, p. 114.

Aubuges, 2, p. 101.

Augmenter, 3, p. $139 ; 4$, p. 210.

Auto, 4, p. 247.

"Auto-refroidi ", 4, p. 210.

Autorité du traducteur sur la profession, 1, p. 72.

Auto-tamponneuse, 3, p. 146.

Auto-transformateur, 4, p. 209.

Avenant, 4, p. 245.

Bac à glaçons, 1, p. 55

Bac à légumes, 1, p. 55.

Baie d'observation, 1, p. 56.

"Baisse », 4, p. 210.

Baladeuse, 2, p. 113.

Balbutiements (en être aux), 3, p. 146.

Ballastage, 1, p. 37.

Banalisée, 1, p. 37.

Banque de terminologie, 4, p. 191.

Batture, 2, p. 109.

"Bénéfice ", 4, p. 245.

Bénéfice de gestion, 4, p. 246.

Bibliophile, 2, p. 94 .

Bibliothécaire adjoint, 2, p. 113.

Bibliothèque orale, 2, p. 88 .

Biennale de Liège, 4, p. 256

Biens d'équipement, 4, p. 238.

Bienveillance, 4, p. 218.

Blancheterre, 2, p. 101

Blanchisserie, 4, p. 212.

Blé d'Inde, 2, p. 109.

Bleuet, 2, p. 109.

Bobinage, 4, p. 210

Bois (dictionnaire du ), 4, p. 248

"Boîtier à glaçons », 1, p. 55 .

Bon (en parlant d'un lac) , 3, p. 143.

Bon vouloir, 4, p. 218.

«Boni », 3, p. 169.

Bonne volonté, 4, p. 218.

Borne, 4, p. 209.

"Break », 1, p. 35.

Bretelle, 2, p. 109.

Bricolage, 2, p. 119.

Buanderie, 4, p. 211.

Buraliste, 2, p. 113

Bureau, 3, p. 159

"Bureau des directives", 2, p. 114.

"Bureau des gouverneurs ", 3, p. 159

Cadre, 3, p. 160

Cafeteria, 2, p. 107. 
Canadianisme, $3, p .180$

Canal expérimental à irradiation, 4, p. 233.

Capacité, 4, p. 209.

Capital-client, 4, p. 218.

Capital-clientèle, 4 , p. 218.

Caractère professionnel des associations, 4 , p. 254.

Caractérisation des noms de marque, 3, p. 178.

Carence, 4, p. 237.

"Casier , 1, p. 55

Catégorie " animés », 4, p. 202.

Caténaire, 1, p. 35.

Champ sémantique, 4, p. 204.

Champignon, 1, p. 37.

Changeur de prises en charge, 4, p. 210.

Chassé-croisé, 4 , p. 227

Chef de pupitre, 2, p. 113

Cheval, 4, p. 248.

Chimie, 1 , p. $59 ; 2$, p. $118 ; 3$, p. 167

Chimie radio-active, 4 , p. 232

Chinois, 3, p. 171.

Chroniqueur, 4, p. 208.

Cinégramme, 2 , p. 113.

"Circuit logique ", 1, p. 37

Civilisation grecque, 4, p. 248.

Civilisation romaine, 3 , p. 171

Clayette, 1, p. 55 .

Clientèle, 4, p. 218.

Climat de confiance, 4 , p. 218

Code régional, 2, p. 114.

Collaborateur, 3, p. 155.

"Collège des médecins », 1, p. 78.

Colloque de la participation, 4, p. 253.

Colloque sur la traduction, 4, p. 257.

Combiné de mesure, 4, p. 211.

Comité, 3, p. 159.

"Comité parlementaire ", 3, p. 159.

Commerce, 2, p. 119.

Commercialisation, 2 , p. 103.

Commis, 4, p. 223.

Commissaire du croire, 2, p. 113.

Commission, 3, p. 159.

"Commission scolaire », 3, p. 159.

Commis-voyageur, 4 , p. 223.

"Commonwealth», 2, p. 109.

Communication, 4, p, 242.

Commutateur, 4, p. 210.

Compétence, 1, p. 72 .

Composition synthétique, 4 , p. 234

Comptabilité, 2, p. 118

Comptabilité hospitalière, 2, p. 119.

"Compte payable ", 3, p. 169.

"Compte recevable", 3, p. 169.

Comptes à payer, 3, p. 169.

Comptes à recevoir, 3, p. 169.

Comptes Clients, 3, p. 169.

Comptes Fournisseurs, 3, p. 169

Comptoir de cuisine, 1 , p. 55

Concentration, 4, p. $227,229$.

Conception publicitaire, 2, p. 96

Conditions de travail du traducteur, 1, p. 73.

Conférence de R. Le Bidois, 4, p. 256.

Configuration, 3 , p. 164

Congélateur, 1, p. 55

Congés payés, 3, p. 169.

Conjoncturel, 4, p. 242.

Conjugaison anglaise, 1, p. 52 .

Connexion en zigzag, 4, p. 210.
Connexion étoile, 4, p. 210.

Connexion triangle, 4 , p. 210

Consécutive (en), 2, p. 84

Conseil, 3, p. 159

Conservateur, 4, p. 209.

"Consistant", 2, p. 110

Construction mécanique, 2, p. 119.

Contamination sémantique des homophones, 3 , p. 178.

Conteneur, 3, p. 161.

Conteneur universel, 3 , p. 161.

Contenter tout le monde et son père, 1, p. 23

Contenu culturel, 4, p. 203.

Contenu lexical, 4, p. 203.

Contestation, 4, p. 237.

Contexte, 1, p. $42 ; 3$, p. 164.

Continental, 3 , p. 161 .

Contrainte, 1 , p. 11.

Contribution des adhérents, 3, p. 156.

Contribution patronale, 3 , p. 156 .

Contrôler, 3, p. 178.

Conversion, 4 , p. 246

"Conversion alimentaire ", 3, p. 168.

"Corporation ", 1, p. 45 .

"Corporation de charité », 1, p. 46.

Correction du français, 4, p. 247.

Correspondance, 1 , p. 60 .

Corse, 1 , p. $58 ; 4$, p. 247.

Cotisation des participants, 3, p. 156.

Cotisation salariale, 3 , p. 156 .

Coudre un bouton, 3 , p. 139.

Couleur, 4 , p. 217.

Coulure, 1, p. 71 .

Coureur des bois, 2, p. 109

Cours magistral, 3 , p. 138 .

Couvercle, 4, p. 210

Craquer, 3, p. 150.

Création française, 3 , p. 177.

Création littéraire, 1, p. 8

Crédit, 3, p. 147

Crème de..., 4, p. 219.

Crèmerie, 3 , p. 168 .

Culture et grammaire, 4, p. 202.

Cuve, 4, p. 209

Dans tous les cas où, 3, p. 149.

Dateur, 2, p. 113.

De suite, 1 , p. 41.

Débarbouillette, 2, p. 109.

Décimer, 2, p. 110

Décision, 4 , p. 242

Défense d'entrer, 3 , p. 136

Défense de déposer des ordures, 1, p. 10.

Définition de la publicité, 3 , p. 179

Demande en justice, 1, p. 43.

Demiard, 2, p. 109

Démonstratif, 3, p. 156.

Déplacement et mouvement, 3, p. 139.

Dépouillement, 4, p. 227.

Désuet, 2, p. 111.

Détecteur par activation, 4, p. 233.

Détruire, 3, p. 142.

Dettes actives, 3, p. 169.

Dettes passives, 3 , p. 169 .

Dictionnaire, 4, p. 247.

Dictionnaire automatique, 1, p. 71; 4, p. 191.

Diélectrique liquide, 4, p. 209.

Diesel, 1, p. 35.

Différentiel, 1, p. 13 
Difficultés de la langue française, 2, p. 117.

Dilution, 4, p. 227, 228.

Diminuer, 4, p. 210

Diriger les trains, 1, p. 37.

Dirigisme de l'Etat, 3, p. 177

Disjoncteur, 4, p. 209.

Dispositif anticheminant, 1, p. 37.

Dites-le avec des fleurs, 3 , p. 178 .

Documentation, 1, p. $71 ; 3$, p. $182 ; 4$, p. 191.

Dominion, 2, p. 109.

Donc, 3, p. 142.

Drave, 2, p. 109

Droit de transformation, 4, p. 246.

Droit et bilinguisme, 4, p. 257.

Dur, 4, p. 197.

Ecart, 3, p. 137

Ecole de publicité, 3, p. 178

Ecole structuraliste, 4, p. 205.

Economie, 1, p. 59; 4, p. 227, 229, 240

Economie lexicale, 2, p. 96.

Ecriture, 1, p. 14, 15 .

Editer, 4, p. 207.

Editeur, 1, p. 31; 4, p. 207.

Edition, 4, p. $207,208$.

Editionner, 4, p. 208.

Editorial, 4, p. 208.

Editorialiste, 4, p. 208.

Electrifier, 3, p. 139.

Electriser, 3, p. 139.

Electronique, 1, p. 59

Electrophone de table, 2, p. 102.

Electrotechnique, 1 , p. $59 ; 4$, p. 240 .

Emballage, 2, p. 116.

Emettre, 4, p. 244

Emonder, 2, p. 97

Employé, 3, p. 154

Emprunt, 2, p. 122

Endossement, 4, p. 244, 245

Energie d'activation, 4, p. 233.

Enroulement, 4, p. 209.

“ Entrée », 2, p. 109.

Enumération (procédé d'écriture), 1, p. 18

Enumération (procédé stylistique), 1, p. 16, 17.

Enuméré, 3, p. 169.

"Environnement ", 1, p. 37; 2, p. 110.

Envoler (s'), 3, p. 139.

Enzyme, 3, p. 178.

Epinette, 2, p. 109.

Epouvantail, 4, p. 197.

Eprouvette soumise à irradiation, 4, p. 233.

Equipement, 4, p. 238.

Equivalence, 3, p. 137, 165 .

Erablière, 2, p. 109.

Ergothérapeute, 2, p. 101.

Essai, 4, p. 210.

Essai d'échauffement, 4, p. 210.

Essai de réception, 4, p. 210

Essai de tenue au choc, 4, p. 210.

Essai de type, 4, p. 210

Essai individuel, 4, p. 210.

Essence * d'arbres », 3, p. 168.

Essoreuse, 1, p. 56.

Ethique professionnelle, 4, p. 240.

Ethnographie sémantique, 4, p. 204.

Ethnoscience, 4, p. 204.

Etoffement, 4, p. 227

"Exécutif , 3, p. 159.

Explicitation, 4, p. 227, 229.
Exposition canine, 2, p. 94.

Farad, 4, p. 209.

Faux amis, 1, p. $70 ; 3$, p. 136, 166, 178.

Ferme, 3, p. 168.

Fidélité, 3, p. 165.

Fleurs, 4, p. 247.

Folklore et publicité, 3, p. 179.

Fondre l'argenterie, 3, p. 139.

Fonds de commerce, 4, p. 218.

Fondue, 4 , p. 220

Foraine, 2, p. 101.

Formation du traducteur, 1, p. 7, 70; 2, p. 98.

Formations négatives, 3, p. 177.

Forme progressive, 2, p. 122.

Formellement interdit, 3 , p. 136.

Fragilisation à hautes températures sous irradiation, 4, p. 233.

Français, 3, p. 170.

Français commercial, 1, p. 60.

Français populaire, 4, p. 249.

Frigorifique, 1, p. 36.

Fromage à la pie, 4, p. 219.

Fromage à tartiner, 4, p. 220.

Fromage blanc, 4, p. 219.

Fromage cottage, 4, p. $219,222$.

Fromage de cottage, 4, p. 219

Fromage fermière, 4, p. 219.

Fromage fondu, 4, p. 219.

"Fromage refait ", 4, p. 220

Galène, 3, p. 147.

Gapan, 2, p. 101.

Garantie, 4, p. 245.

Gastronomie, 2, p. 118.

Gâteau maison, 3, p. 178

Gel de silice, 4, p. 209.

Gerber, 1, p. 37.

Gestion, 4, p. 240.

Girafe, 2, p. 101.

Gouverneur général, 2, p. 109.

"Gradué », 2, p. 110.

Graisse alimentaire, 4, p. 220.

Graisse préparée, 4, p. 220.

Graisse végétale, 4 , p. 220 .

Grammaire, 3, p. 136.

Grammaire comparée, 1 , p. 58.

Grammaire différentielle, 1, p. 70

Grammaire et culture, 4, p. 202.

Grammaire transformationnelle, 4, p. 231.

Grammatical et idiomatique, 3, p. 137.

Grand film, 3, p. 138.

Gratification, 3, p. 169.

Grivèlerie, 2, p. 96.

Groupe de défense du français, 3, p. 168.

Grue, 2, p. 101.

Habitant, 2, p. 109.

Halle automatisée, 3 , p. 161.

Hang up (to), 3, p. 139

"Hausse ", 4, p. 210.

Haut (le) de la pyramide, 3, p. 144.

Haut-le-pied, 1, p. 35.

Hayon élévateur, 3, p. 162.

Histoire, 1, p. 5.

Histoire de l'écriture, 4, p. 250.

Hmong, 3, p. 170.

Hopi, 4, p. 203.

Hublot, 1, p. 55

Huile minérale, 4, p. 209.

Huile végétale, 4, p. 220. 
Hydruration, 4, p. 234.

Hygiène, 2, p. 118 .

Idées clefs, 4, p. 235.

Idiomatique et grammatical, 3, p. 137.

Idolâtrie, 2, p. 97.

Il n'y a rien de tel, 3, p. 137.

Immergé, 4, p. 209.

Implicitation, 4, p. 227, 229.

"Incrément », 2, p. 101

Industrie automobile, 2, p. 123.

Infinitif sans sujet, 3 , p. 178 .

Informatique, 1 , p. 38 .

Inhalothérapie, 1, p. 78

Inhérence, 3, p. 138 .

Insécurité linguistique, 3 , p. 178

Installations fixes, 1 , p. 36 .

Intercontinental, 3 , p. 161 .

Interprétariat, 2, p. 84.

Interprétation, 1, p. $60 ; 2$, p. 83,84 .

Interprète professionnel, $2, \mathrm{p} .83$.

Inversions passives, 1, p. 10 .

Irium, 3, p. 178 .

Isotherme, 1 , p. $36 ; 3$, p. 161

Japonais, 1, p. 60

Je/nous, 1, p. 10.

Je suis payé pour le savoir, 3, p. 137.

Je vous crois, 3, p. 137.

Jersiais, 1 , p. 58 .

Joncteur, 4, p. 233

Journalisme, 2, p. 112

$L$ mouillé, 2 , p. 111.

Là où, 3 , p. 149.

Laine-velours, 3, p. 178

Laisser pénétrer, 3, p. 142.

Laiterie, 3, p. 167.

Langage agricole, 3 , p. 167

Langage pétrolier, 2, p. 123.

Langue administrative, 1, p. 59.

Langue d'arrivée, 1, p. 5.

Langue de départ, 1, p. 5 .

Langue de diffusion, 4, p. 201.

Langue de marché, 4, p. 201.

Langue et pensée, 4, p. 200

Langue internationale, 4 , p. 201.

Langue tchèque, 2, p. 117.

Langues algonquines, 4, p. 202.

Largeur intérieure, 3 , p. 148.

Lecteur de textes, 4, p. 208.

Lecture réflexe, $1, \mathrm{p} .75$.

Législature, 2, p. 109.

Lessiveuse, 4, p. 214.

Lexème, 4, p. 205.

Lexicologie, 3 , p. 136

Lexicologie différentielle, 1, p. 70.

Linge d'hôpital, 4, p. 217.

Linguistique, 2 , p. $119 ; 4$, p. 248

Linguistique allemande, 3 , p. 172.

Linguistique anglaise, 3, p. 172.

Linguistique appliquée, 2 , p. 123

Linguistique bulgare, 1, p. 58 .

Linguistique comparée, 1, p. 58 .

Linguistique différentielle, 1 , p. $70 ; 3$, p. 135.

Linguistique du blanc-russe, 1, p. 58.

Linguistique française, 3 , p. 173 .

Linguistique générale, 3, p. $173 ; 4$, p. 249.

Linguistique polonaise, 1, p. 57.

Linguistique blanc-russe, 1, p. 57.

Linguistique serbo-croate, 1, p. 58
Linguistique slovaque, 1, p. 57.

Linguistique tchèque, 1 , p. 57 .

Linguistique ukrainienne, 1, p. 58

"Listé ", 3, p. 169.

Locomotives de manouvre, 1, p. 35.

Longueurs de la traduction, 2, p. 9.

Machine à additionner, 2, p. 113.

Macrocontexte, 3, p. 164.

Maliens, 2, p. 110

Mandat, 1, p. 46.

Manier l'épée, 3, p. 142.

Manceuvré, 4, p. 210.

Manuels scolaires, 1, p. 32; 4, p. 255.

Manutention, 2, p. 116.

" Marcher au bureau ", 3, p. 139.

Matériel, 2, p. 104.

Matériel agricole, 3, p. 168.

Matériel de traction, 1, p. 35

Matériel marchandises, 1, p. 35.

Matériel polytherme, 3, p. 161

Matériel voyageurs, 1, p. 35 .

Matières plastiques, 2, p. 119.

Mécanisme d'entraînement, 4, p. 210.

Mécanoïde, 2, p. 101, 104.

Médecine du travail, 2, p. 118

Membre adhérent, 3, p. 156.

Membre participant, 3, p. 156

Mémoire à la Commission Gendron, 4, p. 254

Mesure, 2, p. 109.

Mesure de tensions de court-circuit, 4, p. 210

Mesure du rapport de transformation, 4, p. 210.

Métaphore, 4, p. 248.

Méthode de traduction, 1, p. 76 .

Méthodes d'enseignement, 2, p. 83.

Méthodologie de la traduction, 2, p. 83.

Micro de table, 2, p. 102

Microcontexte, 3, p. 164.

Millage, 2, p. 109.

Minéralier, 2, p. 109

Minimum (un) de..., 3, p. 149

Mire de réglage, 4, p. 243.

Mire électronique, 4, p. 243.

Misse1, 1, p. 6.

Modification, 4, p. 246.

Monnaie, 2, p. 109

Monorail, 2, p. 109.

Monosémie, 3, p. 166

Montage, 4, p. 208.

Monter un film, 4, p. 207.

Monteur de film, 4, p. 208.

Montréalais, 2, p. 110.

Morphologie, 4, p. 202.

Motif, 1, p. 38 .

Motiver, 1, p. 38

Motrice panoramique, 1, p. 36.

Mots-valises, 1, p. 61.

"Moulée ", 3, p. 168

Mouvement et déplacement, 3, p. 139.

Musical, 3, p. 138.

Ne... pas, 4 , p. 197

N'entrez pas, 3, p. 136.

Nature des transpositions, 4, p. 230.

"Négocier un virage ", 2, p. 110.

$\mathrm{Ne}$ pas plier, 3, p. 136

Néologie, 3, p. 176

Néologisme, 1 , p. $71 ; 2$, p. $122 ; 4$, p. 191.

Neuf sur dix, 3, p. 152. 
Nigérien, ian, ois, 2, p. 110.

Niveaux de langue, 2, p. 94

Nom " animé ", 4, p. 202.

Nom (le) est Paquette, 3, p. 137.

Normalisation terminologique, 4, p. 191.

Normes quantitatives, 3, p. 157.

Noyau magnétique, 4, p. 209.

Numération globulaire, 2, p. 94

O (joncture savante), 4, p. 233 .

Eilleton, 1, p. 56

Office, 3, p. 160.

Optique, 1, p. 59

Optique structurale, 1, p. 11

Ordo, 2, p. 113.

Ordre des médecins, 1, p. 78

Organisation de la profession, 1 , p. 74

Original, 2, p. 109

Ornithologie, 2, p. 118.

Ouananiche, 2, p. 109.

Outillage, 3, p. 167

Ouvrier, 3, p. 155.

Pantographe, 1, p. 35.

Par, 4, p. 233.

Parachutage, 3, p. 150.

Parachuter, 3, p. 150.

Parasiticides, 3, p. 167.

"Parloir de traite ", 3, p. 168

Participation, 4, p. $245,253$.

Participation aux bénéfices, 4 , p. 245 .

Parties du discours, 4, p. 227.

Pas-de-porte, 4, p. 218.

Passage à niveau, 1, p. 37 .

Patauger, 3, p. 139.

Patin, 1, p. 37.

Patronage, 3 , p. 159

Pause-café, 3 , p. 178

Pauvreté d'une langue, 4, p. 203.

Peintres (dictionnaire des), 4, p. 248.

Pensée et langue, 4, p. 200

Pépinière, 3 , p. 146.

Perche, 2, p. 101

Périgramme, 2, p. 104.

Période de prospérité économique, 2, p. 94.

Personnel, 3, p. 155 .

Perte, 4 , p. 210

Perte à vide, 4 , p. 210

Perte due à la charge, 4, p. 210

Peut être ouvert d'office, 1 , p. 10 .

Philosophie, 3, p. 171.

Physiologie, 2, p. 119

Piastre, 2, p. 109

Pied, 2, p. 109

Pied de table, 2, p. 102

Pierre vive, 2, p. 110.

Pile atomique, 2, p. 94.

Pile couveuse, 4 , p. 232

Pile laboratoire, 4, p. 234

Pilotage sans visibilité, 2, p. 94.

Placage, 3, p. 168.

Plan, 2, p. 97

Plomb, 1, p. 56

Plutonium fatal, 4, p. 232.

Poitrine, 4, p. 199.

Politique, 1, p. $59 ; 4$, p. 243.

Polysémie, 3, p. 166.

Pompe, 4, p. 210

Ponctuation, 3, p. 167.

Portage, 2, p. 109.
Porte-à-porte, 3, p. 161.

Porte-auto, 1, p. 36.

Porter, 3, p. 139.

Porte-tourets, 1, p. 36.

Portiques automoteurs, 1, p. 37.

Pose des traverses, 1, p. 37.

Pouce, 2, p. 109.

Poudrerie, 2, p. 109.

Précautions, 3, p. 143.

Précipitation " de pluie ", 3, p. 168.

Premier-Montréal, 4, p. 208.

Premier-Paris, 4, p. 208

Premiers pas, 3, p. 147.

Primaire, 4, p. 210.

Prime, 3, p. 169

Prime au rendement, 3, p. 169.

Priorité du sens, 3, p. 165

Prises, 4, p. 210.

Prix de présence, 2, p. 94

"Procédure », 1, p. 37.

Produit blanc, 4, p. 221.

Profession, 4, p. 236.

Programmoide, 2, p. 101, 104.

\& Projecteur en diapositif 》, 1, p. 56.

Projecteur par transparence, 1, p. 56.

Prosodique, 3 , p. 138.

Province, 2, p. 109.

Publication de traductions, 1, p. 31

Publicité, 2, p. 96; 3, p. 176; 4, p. 242.

Puissance fatale, 4 , p. 232.

Puissance nominale, 4 , p. 209.

Pupitre, 1, p. 56.

Pupitre de mixage, 2, p. 102.

Pyralène, 4, p. 209.

Pyranol, 4, p. 209

Qualités requises d'un traducteur, 3, p. 157.

Québécois, 2, p. 110.

Quote-part répartissable, 4, p. 246.

Radio-, 4, p. 232.

Radio de table, 2, p. 102

Radio-chimie, 4, p. 232.

Rail, 1, p. 34

Rail-route, 1, p. 36.

Rapport de transformation, 4, p. 210.

Réacteur à circulation forcée, 4, p. 234

Réacteur à coeur à germes, 4, p. 234.

Réacteur à cceur fermé, 4, p. 234.

Réacteur à combustible pulvérulent, 4, p. 234.

Réacteur à eau, 4, p. 234.

Réacteur à eau légère, 4, p. 234.

Réacteur à graphite, 4, p. 234.

Réacteur à plutonium, 4, p. 234

Réacteur à sels fondus, 4, p. 234.

Réacteur à surchauffe, 4, p. 234.

Réacteur à tubes de force, 4, p. 234.

Réacteur chaudière, 4, p. 234.

Réacteur d'entraînement, 4, p. 234

Réacteur d'irradiation, 4, p. 233, 234.

Réacteur de production, 4, p. 234

Réacteur de puissance nulle, 4, p. 234

Réacteur piscine, 4, p. 234

Réacteur source, 4, p. 234.

Réacteur surrégénérateur, 4, p. 234.

Réaliser, 3, p. 178.

Récepteur, 3, p. 165

Récepteur de table, 2, p. 102.

"Réception 》, 4, p. 210.

Recherche, 3, p. 147. 
Recherche terminologique, 4, p. 191.

"Réclamation ", 4, p. 245.

Recouvrement des notions, 3, p. 166

Rédacteur en chef, 4, p. 208.

Rédiger, 4, p. 207.

Réfrigérant, 1, p. 36.

Refroidissement, 4, p. 210.

Régie, 3, p. 160.

Régie de studio, 2, p. 102

Régime de prévoyance d'entreprise, 3, p. 156

Régime de prévoyance de personnel, 3, p. 156.

Régime de rente d'invalidité, 3, p. 169.

Régime de rente de pension de retraite, 3, p. 169.

Rejet, 4, p. 210

Relation, 3, p. 138

Relations publiques, 4 , p. 218.

Relaxe, 3, p. 178

Remiser, 1, p. 37

Remorque rail-route, 1, p. 36 .

Rémunération de la traduction littéraire, 1 , p. 32.

Représentant, 4, p. 223.

Résonner, 3, p. 142.

Respirateur, 4, p. 209

Revendicatif, 4, p. 237.

Revendication, 4, p. 236.

Réversible, 1, p. 36.

Reviser, 4, p. 207.

Reviseur, 4, p. 208.

Révision, 2, p. 98.

Rièze, 2, p. 101.

Ristourne, 4, p. 246

"Roadster", 1 , p. 35

"Roc solide », 2, p. 110.

Rotation du personnel, 3, p. 169.

Roue à boudins, 1, p. 34 .

Rupture de charge, 3 , p. 161

Saindoux artificiel, 4, p. 220

Salaire au rendement, 3, p. 169.

Salaison, 3, p. 168

Salarié, 3, p. 155.

"Salon de traite », 3, p. 168

Savon, 4, p. 215.

Sciences économiques, 4, p. 240.

Séance (la) est ouverte, 3 , p. 136.

Sécheuse, 1, p. 56.

Secondaire, 4, p. 210

Sécurité contre le vol, 3, p. 143.

Sécurité du travail, 2, p. 118

Sécurité sociale, 4, p. 243.

Sélectionner, 4, p. 207.

Sens affectif, 3, p. 138.

Sens figuré, 3, p. 138.

Sens intellectuel, 3, p. 138.

Sens interdit, 3 , p. 136.

Sens propre, 3, p. 138.

Sens unique, 3 , p. 136 .

Série transposée, 4 , p. 227

Servitude grammaticale, 4, p. 231.

Seul (un) trait de plume, 3, p. 145 .

Sigles, 3, p. 167

Significatif du style, 3, p. 165.

Simili-saindoux, 4, p. 221.

Simultanée (en), 2, p. 86.

Sinistre, 4, p. 245.

Situation du traducteur, 1, p. 69.

Situations culturelles, 3, p. 178.
Slip, 3, p. 162.

Slogans, 3, p. 180.

"Snack-bar", 2, p. 107

Société, 1, p. 45 .

Société protectrice des animaux, 2, p. 97

Socle de table, 2, p. 102.

Solution " deux portes ", 3, p. 141.

Sonothèque, 2, p. 88.

Sorti des rangs, 3 , p. 150

Soupape de sûreté, 4, p. 209.

Source fatale, 4, p. 232.

"Sous-gradués", 2, p. 110

Spire, 4, p. 210.

Statut du traducteur, 1, p. 69; 3, p. 158.

Statut international du traducteur, 1, p. 75 .

Stimulus stylistique, 3, p. 163.

Stratégie commerciale, 2, p. 103.

"Strictement prohibé ", 3, p. 136

Structuralisme, 4, p. 205.

Structure du vocabulaire, 4, p. 204

Style, 1, p. 14, 15.

Stylistique, 1, p. 14,$15 ; 3$, p. 163 .

Stylistique comparée, 1 , p. $12 ; 3$, p. $136 ; 4$, p. 250.

Stylistique différentielle, 1, p. 70.

Stylistique interne, 1, p. 12

Substitution, 4, p. 227.

Suite, 1, p. 41 .

Super, 2, p. 122.

"Supporter ", 2, p. 97.

Suppression de la préposition, 3 , p. 178.

Surchauffé, 3, p. 143.

Surgénérateur, 4, p. 232.

Surplus, 4, p. 245.

Surpression, 4, p. 209.

Surrégénérateur, 4, p. 232

Susdit, 3, p. 143.

Suspendre, 3, p. 139.

Symboles, 3, p. 167.

Synapsies, 4, p. 232.

Synonymie, 3, p. 166.

Syntagmatique, 4, p. 232.

Syntaxe française, 4, p. 249

Synthème, 4, p. 232.

Système de salaires stimulants, 3, p. 169

Système esthétique individuel, 1, p. 12

Système linguistique, 1, p. 12 .

Système prosodique, 1 , p. 12 .

Système stylistique, 1, p. 12 .

Table, 1, p. 56; 2, p. 101 .

Table d'écoute, 2, p. 102

Table de bruitage, 2, p. 102.

Table de mixage, 2, p. 102

Table de travail, 1, p. 55 .

Table de trucage, 2, p. 102

"Table tournante ", 2, p. 102

"Tablette ", 1, p. 55.

Talon d'une clavette, 3, p. 166.

Tantième, 4, p. 246.

"Tapper un téléphone ", 2, p. 102.

"Tapper une conversation téléphonique ", 2 , p. 102.

"Taux de virement de la main-d'œuvre ", 3, p. 169.

Techniques commerciales, 2, p. 103.

Télécommunications, 2, p. 118 .

Téléphone, 2, p. 118.

Téléscripteur, 2, p. 113. 
Télétype, 2, p. 113.

Téléviseur de table, 2, p. 102.

Télévision, 4 , p. 247

Télex, 2, p. 109.

Témoin, 1, p. 44

* Terme d'office ", 1, p. 46

Terme générique, 4, p. 204

Terme spécifique, 4, p. 204

Termes ferroviaires, 2, p. 115.

Termes juridiques, 2 , p. 115

Terminologie, 3, p. 182; 4, p. 191.

Terminologie comparée, 4, p. 231

Terminologie comptable, 2, p. 122.

Terminologie des transports, 3, p. 160.

Terminologie économique, 4, p. 242.

Terminologie médicale, 1 , p. 78; 4, p. 243.

Terminologie multilingue, 4 , p. 232.

Terminologie saussurienne, 3 , p. 171.

“Terre de surface $», 3$, p. 168

Terre végétale, $3, \mathrm{p} .168$.

Territoire, 2, p. 109.

Tertiaire, 4, p. 210

Textile, 3, p. 168

Thèmes publicitaires, $3, \mathrm{p} .177$

Théorie des décisions, 4 , p. 242

Thérapie inhalatoire, 1 , p. 78 .

Titulaires de revenus modestes, 3, p. 155.

Tombereau, 1, p. 36

"Toupie »1, p. 56.

Tour passif, 4, p. 233.

"Tourbe ", 3, p. 168.

Tourbillon d'air, 3, p. 143.

Tourisme, 1, p. 60.

Tourne-disques, 2, p. 102

Tout de suite, 1, p. 41

Tradition culturelle, 1, p. 12.

Traduction, 1, p. 60

Traduction à vue, 2, p. 87 .

Traduction automatique, 1, p. 62,$65 ; 2$, p. 119 , $129 ; 3$, p. 171

Traduction consécutive, 2, p. 84

Traduction et linguistique, 4, p. 232.

Traduction littéraire, 1, p. $3-33 ; 3$, p. 172.

Traduction orale (phases), 2 , p. 85 .

Traduction publicitaire, 1 , p. 70,$76 ; 2$, p. 96.

Traduction raisonnée, 3 , p. 135.

Traduction scientifigue, 3, p. 166

Traduction simultanée, 2, p. 84.

Traduction technique, 3 , p. $166 ; 4$, p. 231

Traduction théâtrale, 1, p. 77

Traductions impersonnelles, 1, p. 10.

Traductions « personnalisées », 1, p. 10 .

Trafic, 3, p. $178 ; 4$, p. 239

Traîne sauvage, 2, p. 109 .

Traitement, 4, p. 257.

Traitement de l'information, 4, p. 191.

Transcontinental, 3, p. 161.

Transfert, 3, p. 166.

Transformateur, 4, p. 208.

Transformateur à colonnes, 4, p. 209

Transformateur cuirassé, 4, p. 209.
Transformateur de courant, 4, p. 211.

Transformateur de mesure, 4, p. 211.

Transformateur de puissance, 4, p. 209.

Transformateur de tension, 4, p. 211.

Transformateur sec, 4, p. 209.

Transformation, 4, p. 246.

Transformer (se), 3, p. 145.

Transistor, 2, p. 109.

Transpalette, 3 , p. 161.

Transport, 2, p. $115 ; 3$, p. $160 ; 4$, p. 242.

Transport de l'énergie, 4, p. 209.

Transport universel, 3 , p. 161.

Transposition, 2, p. 95; 4, p. 226.

Transroulage, 3 , p. 161.

Travaux (de voirie), 3 , p. 144

"Traverse de chemin de fer $», 1$, p. 37

Traversée, 4, p. 209

"Tribus détruites », 2, p. 110.

Trombones, 2, p. 114

Tu, vous, 1, p. 6 .

Tube de comptage, 4, p. 233.

Tubulure de déversement, 4, p. 209.

Types de transposition, 4, p. 227.

Typhoïde, 3, p. 144.

Typonymie, 2, p. 122

Tyrannie de la forme, 3, p. 140

Ultrason, 1, p. 59.

Unité stylistique, 3 , p. 164

Unités, 3, p. 167.

Unités de traduction, 3, p. 137.

Universel, 3, p. 161

Université Harvard, 2, p. 110.

Usé, 3, p. 139.

Utilisation des dictionnaires, $1, \mathrm{p} .76$.

Vacances annuelles, 3, p. 169.

Ventilateur, 4, p. 210.

Verbe anglais, 2, p. 122

Vocabulaire de la buanderie, 4, p. 211

Vocabulaire médical, 3, p. 171.

Vocation, 4, p. 235.

"Voir à ", 2, p. 109

Voiture, 1, p. $35 ; 4$, p. 222.

Voiture-coach, 1, p. 35.

Voiture-lit, 1, p. 35.

"Votre passeport pour mieux voyager ", 1 p. 10.

Vous, tu, 1, p. 6

Vous ne m'apprenez rien, 3, p. 137

Voyageur, 2, p. 109.

Voyageur de commerce, 4, p. 223

Wagon, 1, p. $35 ; 4$, p. 222.

Wagon couvert à porte encastrée, 1, p. 36 .

Wagon plat, 1, p. 36

"Wagon-lit ", 1, p. 35.

Wagon-poche, 1, p. 36 .

Wagons à parois de bout en $\mathrm{U}, 1, \mathrm{p}, 36$.

Wagons à ranchers, 1, p. 36.

Wagons porte-autos à deux ponts, 1, p. 36 .

Wagon-trémie, 1, p. 36.

Yod, 2, p. 111. 